$\begin{array}{ll}\text { MARTIN SCOTT } & \text { (NOTTINGHAM UNIVERSITY HOSPITALS NHS TRUST) } \\ \text { MURRAY D SMITH } & \text { (UNIVERSITY OF LINCOLN) }\end{array}$

\title{
WHAT IS THE VALUE OF SPECIALIST SHOULDER PHYSIOTHERAPY? A COST- UTILITY ANALYSIS
}

We have evaluated the cost-effectiveness of specialist shoulder physiotherapy in routine practice. When commissioning health services, it is helpful to understand the costs and benefits associated with treatments. Well established generic measures such as the EuroQol (EQ-5D) can be used to estimate health-related quality of life (QALY) thus identifying the benefits of treatment derived by the patient.

EQ-5D responses pre- \& post-treatment were obtained from a sample of $n=142$ patients undergoing routine physiotherapy treatment at a specialist Shoulder Unit over the period 2011-17. Patients were discharged from treatment when symptomfree. Health-related utility scores were calculated using the UK tariff and QALYs were calculated by the area under the curve method (Manca 2005). Simplified treatment costs were calculated from the NHS perspective and assigned using event-weighted average of outpatient physiotherapy attendances reported in NHS Reference Costs 2014-15. The comparator was no treatment, incurring zero treatment cost and no QALY gain assumed.

In the sample: 69 male, 73 female; mean age 35.7 years ( $S D=18.9)$; duration of symptoms averaged 4.9 years; mean number of treatments $5(\max =20)$ over a period averaging 6.7 months ( $\max =2.6$ years). $76 / 142$ had a prior course of physiotherapy for the same complaint. 59 had dominant arm problems, 20 were bilateral. There were 17 different diagnoses, with most patients having atraumatic glenohumeral instability or myofascial tightness of the upper quadrant.

Treatment costs averaged $£ 297$ per patient $(S D=186)$. Mean utility pre-treatment $0.64(S D=0.23)$ differed significantly the mean post-treatment $0.79(S D=0.23)$ paired $t=8.8 p<0.01$. Incremental Cost-Effectiveness Ratio (ICER) $f 774$ per QALY gain.

NICE guidelines suggest that an ICER below $f 20000 / Q A L Y$ is indicative of cost effectiveness within the NHS. Subject to our assumptions, the results imply that specialist shoulder physiotherapy may provide the NHS with excellent value for money, even for patients with long-standing problems who have previously failed to benefit from physiotherapy. 